\title{
Prevalência do aleitamento materno no Distrito Federal, Brasil
}

\author{
Prevalence of breast-feeding \\ in Brasilia, Brazil
}

Maria Cristina Ferreira Sena 1

Eduardo Freitas da Silva 2

Maurício G. Pereira 3

\footnotetext{
1 Coordenação de Pesquisa e Comunicação Científica, Fundação de Ensino e Pesquisa em Ciências da Saúde, Secretaria de Estado de Saúde do Distrito Federal. C. P. 04472, Brasília, DF 70919-970, Brasil.

2 Departamento de Estatística, Instituto de Ciências Exatas, Universidade de Brasília. Campus Universitário Darcy Ribeiro,

Asa Norte, Brasília, DF 70910-900, Brasil.

3 Departamento de Saúde Coletiva, Faculdade de Ciências da Saúde Universidade de Brasília. Campus Universitário Darcy Ribeiro, Asa Norte, C. P. 4370 , Brasília, DF 70910-900, Brasil.
}

\begin{abstract}
This article presents the findings of a population-based cross-sectional survey carried out during a mass immunization campaign. A group of 3,305 mothers whose babies were 0 to 180 days old was selected by systematic sampling and interviewed with a pretested questionnaire. The preval ence of exclusive breast-feeding among 0 - 15 day-old chil dren was $62 \%$ (IC 95\%: 58.5-65.3) and $12.8 \%$ (11.0-14.7), among 151-180 day-old children. The average duration of exclusive breast-feeding was 39.4 days. The preval ence of predominant breast-feeding was invariable along the age intervals showing a mean of 33.1\% (31.4-34.7). The prevalence of partial breast-feeding among 0 - 15 day-old children was $4.5 \%$ (3.1-6.4) and $28.4 \%$ (25.8-31.0) for the mentioned age groups. For maternal breast-feeding, the overall prevalence varied from $96.8 \%$ (95.8-97.7) to 70.9\% (67.7-73.9). In conclusion, although the overall preval ence of breast-feeding in Brasilia is relatively high, the period that children are exclusively breast-fed is short.
\end{abstract}

Key words Breast Feeding; Cross-Sectional Studies; Epidemiology; Child Health

Resumo O artigo relata os resultados de um inquérito transversal, de base populacional, realizado em Dia Nacional deVacinação. Um grupo de 3.305 mães, com filhos na faixa etária de 0 a 180 dias, seleci onado por amostragem sistemática, foi entrevistado com questionário pré-testado. A prevalência do aleitamento materno exclusivo, na faixa etária de 0-15 dias, foi de 62\% (intervalo de confiança de $95 \%$ : 58,5-65,3) e de $12,8 \%(11,0-14,7)$, na faixa etária de $151-180$ dias. A duração mediana do aleitamento materno exclusi vo correspondeu a 39,4 dias. O aleitamento predomi nante manteve-se constante ao longo dos intervalos etários, com uma prevalência média de $33,1 \%(31,4-34,7)$, enquanto o parcial variou de $4,5 \%(3,1-6,4)$ a $28,4 \%(25,8-31,0)$, nas idades mencionadas. Para o al eitamento materno (todas as modalidades), a prevalência foi de 96,8\% (95,8-97,7) e 70,9\% (67,7-73,9), respectivamente. Em conclusão, a preval ência do aleitamento materno no Distrito Federal é el evada, mas é curto o período em que as crianças são amamentadas exclusi vamente ao seio.

Palavras-chave Aleitamento Materno; Estudos Transversai s; Epi demiologia; Saúde da Criança 


\section{Introdução}

Evidências científicas têm constantemente apontado os benefícios do aleitamento materno para a saúde infantil, principalmente do nascimento até 4 a 6 meses de idade (Cunningham, 1981; Feacham \& Koblinsky, 1984; Habicht et al., 1986; Victora et al., 1987; Yoon et al., 1996). Entretanto, para determinados segmentos populacionais, essa prática alimentar é pouco freqüente. A baixa prevalência do aleitamento materno, sobretudo nos grupos sociais menos favorecidos, assume características de importante problema de saúde pública.

Visando garantir a melhoria das condições de vida do grupo infantil, governos e sociedade civil, com a cooperação de agências internacionais, como a OPAS (Organização Pan-Americana da Saúde) e o U NICEF (Fundo das Nações Unidas para Infância), têm se mobilizado, definindo prioridades, destinando recursose estabelecendo metas para a saúde da criança. Entre essas metas, encontra-se a de “...promover o aumento mínimo de $30 \%$, em relação aos valores da década de 80, nos índices de al ei tamento materno exclusivo nos primeiros seis meses de vida e o início adequado da alimentação complementar, mantendo o lei te materno até dois anos deidade..." (MS, 1994:10). Nesse quadro, torna-se importante a realização periódica de estudos, de base populacional, que informem a freqüência da amamentação nas populações e em determinados grupos sociais, permitindo acompanhar as tendências do al eitamento natural e suas modalidades, nas localidades investigadas.

São poucas as publicações sobre investigações de base populacional para acompanhar as tendências do aleitamento materno nos países em desenvolvimento (Horta, 1996; Leão, 1992; Monteiro, 1988; Notzon, 1984). Fatores de natureza diversa contribuem para esse quadro, dentre eles, as dificuldades próprias da metodologia de investigação. Um dos principais problemas que dificulta a realização de inquéritos populacionais de prevalência é a obtenção de uma amostra representativa. Pesquisas institucionais excepcional mente reúnem um grupo de elementos que retrate a população da região na qual está localizado o serviço. A alternativa de investigação domiciliar é trabalhosa, demorada e tem custo el evado. Uma opção que tem sido tentada, pois facilita o planejamento e a coleta dos dados, é o inquérito ser realizado entre mães que comparecem ao postos de vacinação no Dia Nacional de Multivacinação, dado que tais campanhas usualmente apresentam cobertura elevada.
Não é do nosso conhecimento a existência, no Distrito Federal, de pesquisa de base populacional sobre o tema. Diante dessa constatação, realizamos uma investigação em crianças de 0 a 6 meses de idade, em dia de multivacinação. O presente artigo apresenta as prevalências estimadas para o al eitamento materno (todas as formas), para suas modalidades exclusivo, predominante e parcial, e a estimativa da duração mediana da amamentação exclusiva.

\section{Material e método}

\section{Tipo de estudo}

Foi feito um estudo transversal de base populacional, em amostra de crianças que compareceram aos postos urbanos de vacinação no decorrer da primeira etapa da Campanha Nacional deVacinação, em 11 de junho de 1994. A população do Distrito Federal é 95\% urbana. A campanha é uma estratégia adotada pelo $\mathrm{Mi}$ nistério da Saúde (MS), a partir de 1980, inicialmente, com o objetivo de erradicar a poliomielite por meio da aplicação da vacina oral em todas as crianças menores de cinco anos. Posteriormente, foi ampliada com a administração de outras vacinas. É realizada, anualmente, em duas etapas e, ao mesmo tempo, em todas as unidades da federação.

\section{Amostra}

A população-alvo do estudo compreende crianças, nascidas no período de 11 de dezembro de 1993 a 11 de junho de 1994, residentes no Distrito Federal e presentes nos postos urbanos de vacinação de todas as regiões administrativas. O tamanho dessa população foi determinado a partir do número de menores de um ano e da cobertura vacinal de campanhas anteriores. Assim, estimou-se que, em 1994, aproximadamente 46 mil crianças teriam menos de um ano e, nesse grupo, em torno de 50\% (23 mil) teriam idade igual ou menor que 180 dias. Com base nas coberturas vacinais das campanhas de 1990 a 1993, que variaram de $89 \%$ a 99\% (Departamento de Saúde Pública da Secretaria de Saúde do Distrito Federal, dados não publicados), supôs-se, de forma conservadora, que $80 \%$ seriam vacinadas no dia da pesquisa, o que corresponderia a uma população-alvo de 18.400 crianças.

O tamanho da amostra foi estimado em $20 \%$ da população-alvo (18.400 crianças), o que equivale a 3.680 crianças. Optou-se por esse percentual, em face da preocupação de ga- 
rantir a representatividade dos menores de trinta dias, visto que, por ser esse grupo muito jovem, as mães poderiam apresentar alguma resistência em vaciná-los durante a multivacinação. As crianças foram selecionadas de modo sistemático, uma vez que já estavam ordenadas em fila e o posicionamento das mesmas deu-se aleatoriamente, conforme a chegada ao posto de vacinação. O intervalo amostral, calculado a partir da população-alvo e do tamanho da amostra, foi de 5. Desse modo, o entrevistador identificava grupos de cinco crianças, com idade para o estudo, e escolhia a última para compor a amostra. Caso essa criança não estivesse acompanhada da mãe, prosseguia-se a busca na fila até encontrar uma outra, com as características mencionadas, também acompanhada da mãe.

Na campanha de vacinação em que foi realizado o inquérito, foram instalados 222 postos urbanos. A pesquisa foi delineada para incluir todos os postos, por instâncias dos técnicos da Secretaria da Saúde, que desejavam as informações por região administrativa. Em um dos postos, porém, o inquérito não foi feito devido à ausência dos entrevistadores e, em cinco outros, houve o extravio dos questionários. Nos demais 216 , a pesquisa transcorreu sem anormalidades. No trabalho de campo, houve a participação de 49 supervisores e 503 entrevistadores, o que correspondeu, em média, a um supervisor para cada quatro postos e para cada dez entrevistadores. Nesses 216 postos, a população amostrada foi de 3.305 crianças, representando $18 \%$ da população-alvo estimada para o inquérito (18.400) e 89,8\% do tamanho da amostra previsto para a investigação (3.680 crianças). A diferença entre o número de crianças calculado para compor a amostra (3.680) e as efetivamente pesquisadas (3.305), foi de 375 (10,2\%). Essa diferença pode ter ocorrido devido ao já mencionado extravio de alguns questionários, às crianças identificadas na fila mas não selecionadas para a amostra porque não estavam acompanhadas das mães, a não realização do inquérito em um posto por ausência do entrevistador e, finalmente, por erros nas estimativas.

Na seleção dos questionários válidos, fez-se uma crítica aos mesmos, com base em uma matriz construída a partir das questões referentes à introdução de outros alimentos e utensílios empregados na sua administração. Dessa forma, foi verificada a consistência entre essas informações e os tipos de aleitamento definidos para o estudo, eliminando-se da análise de prevalência, aqueles que apresentavam inconsistências em relação a esses itens.
Dos questionários respondidos, 3.104 (93,9\%) foram considerados válidos para a análise de prevalência. A descrição da amostra do presente estudo foi feita com base em 2.983 (90,3\%) questionários, que estavam livres de erros de preenchimento nas demais variáveis descritivas. As localidades foram agrupadas, conforme características socio-econômicas, em regiões mais e menos desenvolvidas.

\section{Coleta de dados}

O questionário utilizado divide-se em três partes, referentes respectivamente, aos dados relativos à identificação da criança na fila, à criança selecionada para a entrevista e às características da mãe.

As perguntas são fechadas e o número de opções de resposta varia de dois a seis. Para algumas, as respostas são mutuamente exclusivas e para outras, podem ser assinaladas mais de uma alternativa. Utilizou-se a linguagem coloquial na construção de frases curtas e diretas. Não há perguntas hipotéticas sobre preferências, comparações e opiniões. Os quesitos sobre o aleitamento materno abordaram a situação das crianças da amostra em relação a essa prática, à época da pesquisa, o que em inglês, é denominado current breast-feeding status (Notzon, 1984).

A preparação do questionário envolveu a elaboração de sete versões. Nas cinco primeiras, participaram a coordenadora da pesquisa e técnicos da OPAS e do Programa Nacional de Incentivo ao Aleitamento Materno, do MS. Na seqüência, aplicaram-se as versões 6 e 7 em três unidades de saúde. O teste dessas versões não ocorreu em condições idênticas às da pesquisa, uma vez que aconteceu durante a rotina de trabalho desses serviços.

Acompanhava o questionário um manual, que foi utilizado como material instrucional no treinamento dos supervisores e entrevistadores, e como referencial de consulta no dia do inquérito.

Os supervisores do trabalho de campo (pediatras, enfermeiras, nutricionistas e odontólogos) foram treinados pela coordenadora da pesquisa, na metodologia proposta. Participaram como entrevistadores, alunos do segundo grau das escolas públicas do Distrito Federal, submetidos a treinamento ministrado pelos supervisores, no decurso da semana que antecedeu o estudo. A distribuição dos entrevistadores pelos postos de vacinação teve como critério a proximidade com o respectivo domicílio.

$\mathrm{O}$ aleitamento materno e seus tipos foram definidos nos seguintes termos (UNICEF et al., 1991; WHO/ UNICEF, 1992): 
- Aleitamento materno: crianças alimentadas no seio materno, subdividido em três categorias:

a) aleitamento exclusivo: crianças alimentadas somente no seio materno, sem uso de água, chás e sucos;

b) aleitamento predominante: crianças alimentadas no seio materno, às quais foram administradas, adicionalmente, água, chá e suco, isoladamente ou associados;

c) aleitamento parcial: crianças alimentadas no seio materno e às quais já era administrado o leite de vaca, independentemente da introdução de água, chá, suco ou cereais;

- Aleitamento artificial: crianças que não estavam sendo alimentadas no seio materno.

\section{Análise estatística}

A análise estatística foi feita de forma a estimar a prevalência do aleitamento materno e de suas modalidades, por ponto e por intervalo, com $95 \%$ de confiança. Calculou-se também, a duração mediana da amamentação exclusiva. Embora a prevalência do aleitamento materno possa ser estimada pela soma das prevalências de suas modalidades (exclusivo, predominante e parcial), optou-se pela aplicação do modelo probito, dado o interesse em obter a medida por intervalo. Sobre o aleitamento parcial, procedeu-se também a transformação logaritmo neperiano da variável idade da criança. A adequação do ajustamento de cada uma das funções empregadas nas estimativas de prevalência foi verificada pela "razão de máxima verossimilhança", de Wilks (Agresti, 1990). Todas as estimativas foram feitas por ponto e por intervalo, sendo o grau de confiança estabelecido em $95 \%$ (IC 95\%). O programa usado na análise dos dados foi o SAS (SAS Institute, 1990).

\section{Resultados}

As características da amostra estão na Tabela 1 e sugerem que ela é representativa. Observa-se um discreto predomínio de crianças do sexo masculino, e todos os intervalos etários estão adequadamente constituídos para gerar estimativas de prevalências com precisão (ou seja, pequeno intervalo de confiança). A amostra foi separada em dois grupos, segundo o local de residência. A distribuição das variáveis esteve dentro do esperado: nas regiões mais desenvolvidas, as mães eram mais velhas, tinham maior escolaridade e maior proporção de partos cesáreos.

As estimativas de prevalência dos tipos de aleitamento estão na Figura 1. A tendência é decrescente para o aleitamento exclusivo e ascendente para o parcial. A Tabela 2, fornece os valores da prevalência pontual e dos respectivos intervalos de confiança para o aleitamento materno e suas modalidades, exclusivo e parcial, segundo faixa etária. As estimativas para aleitamento materno (todas as formas) variaram de $96,8 \%$ a $70,9 \%$ ao longo dos intervalos etários. A prevalência do aleitamento exclusivo decresceu de $62 \%$ para $12,8 \%$, enquanto que para o parcial variou de $4,5 \%$ a $28,4 \%$. Para o predominante, a prevalência foi de $33,1 \%$, constante ao longo de todas as faixas de idade.

A Tabela 3, mostra os dados a partir dos quais foram estimadas as prevalências da amamentação na amostra investigada. As equações de regressão para cada uma das modalidades de aleitamento estudadas, assim como as respectivas estatísticas e graus de liberdade, podem ser obtidas junto aos autores.

A utilização da transformação logito, para o cálculo da prevalência do aleitamento exclusivo, permitiu-nos estimar a duração mediana dessa forma de amamentação, que foi de 39,4 dias.

\section{Discussão}

Os dados da pesquisa mostram o quadro de base para comparações futuras da amamentação no Distrito Federal. Entre os achados encontrase uma alta prevalência do aleitamento materno (todas as formas), de $70,9 \%$ nas crianças com 151-180 dias de vida, e uma baixa duração mediana do aleitamento exclusivo (39,4 dias).

A presente investigação, tem como aspectos positivos o seu planejamento e execução, conduzidos de modo a evitar vieses de amostragem e de aferição das informações. Tais aspectos, realçados a seguir, indicam que a amostra selecionada é de bom tamanho e representativa da população do estudo, e que os dados foram adequadamente coletados. Por conseguinte, os resultados da pesquisa sobre o aleitamento materno, aqui relatados, refletem a real situação dessa prática alimentar no Distrito Federal, na época investigada.

Utilizou-se a amostragem sistemática, técnica habitual mente empregada quando a população a ser pesquisada vai se formando no decorrer da investigação e a ordenação de seus elementos dá-se ao acaso (Cochram, 1965), como parece ter sido o caso deste inquérito. A magnitude das perdas foi pequena, menos de $10 \%$. Quanto à natureza dessas perdas, as análises sugerem que elas foram al eatórias. A distribuição por sexo mostrou um número ligeira- 
Distribuição das variáveis sócio-demográficas investigadas na amostra de crianças com idade igual ou menor que 180 dias, segundo a região de moradia. Distrito Federal, 1994.

\begin{tabular}{|c|c|c|c|c|c|c|}
\hline \multirow[t]{2}{*}{ Variáveis } & \multicolumn{2}{|c|}{$\begin{array}{l}\text { Regiões mais } \\
\text { desenvolvidas } 1\end{array}$} & \multicolumn{2}{|c|}{$\begin{array}{l}\text { Regiões menos } \\
\text { desenvolvidas }\end{array}$} & \multicolumn{2}{|c|}{ Total } \\
\hline & $\mathrm{n}$ & $\%$ & $n$ & $\%$ & $n$ & $\%$ \\
\hline \multicolumn{7}{|c|}{ Sexo da criança } \\
\hline Masculino & 412 & 50,7 & 1.092 & 50,3 & 1.504 & 50,4 \\
\hline Feminino & 400 & 49,3 & 1.079 & 49,7 & 1.479 & 49,6 \\
\hline Total & 812 & 100,0 & 2.171 & 100,0 & 2.983 & 100,0 \\
\hline \multicolumn{7}{|c|}{ Idade da criança (dias) } \\
\hline $0-15$ & 40 & 4,9 & 127 & 5,8 & 167 & 5,6 \\
\hline $16-30$ & 73 & 9,0 & 193 & 8,9 & 266 & 8,9 \\
\hline $31-60$ & 159 & 19,6 & 382 & 17,6 & 541 & 18,1 \\
\hline $61-90$ & 146 & 18,0 & 375 & 17,3 & 521 & 17,5 \\
\hline $91-120$ & 141 & 17,4 & 399 & 18,4 & 540 & 18,1 \\
\hline $121-150$ & 143 & 17,6 & 351 & 16,2 & 494 & 16,6 \\
\hline $151-180$ & 110 & 13,5 & 344 & 15,8 & 454 & 15,2 \\
\hline Total & 812 & 100,0 & 2.171 & 100,0 & 2.983 & 100,0 \\
\hline \multicolumn{7}{|c|}{ Idade materna (anos) } \\
\hline$\leq 19$ & 94 & 11,6 & 420 & 19,3 & 514 & 17,2 \\
\hline $20-29$ & 503 & 61,9 & 1.352 & 62,3 & 1.855 & 62,2 \\
\hline $30-39$ & 204 & 25,1 & 381 & 17,5 & 585 & 19,6 \\
\hline$\geq 40$ & 11 & 1,4 & 18 & 0,9 & 29 & 1,0 \\
\hline Total & 812 & 100,0 & 2.171 & 100,0 & 2.983 & 100,0 \\
\hline \multicolumn{7}{|c|}{ Escolaridade materna (anos) } \\
\hline 0 & 18 & 2,2 & 99 & 4,6 & 117 & 3,9 \\
\hline $1-8$ & 276 & 34,0 & 1.393 & 64,2 & 1.669 & 56,0 \\
\hline $9-11$ & 330 & 40,6 & 636 & 29,2 & 966 & 32,4 \\
\hline$\geq 12$ & 188 & 23,2 & 43 & 2,0 & 231 & 7,7 \\
\hline Total & 812 & 100,0 & 2.171 & 100,0 & 2.983 & 100,0 \\
\hline \multicolumn{7}{|c|}{ Parto cesariano } \\
\hline Sim & 410 & 50,5 & 601 & 27,7 & 1.011 & 33,9 \\
\hline Não & 402 & 49,5 & 1.570 & 72,3 & 1.972 & 66,1 \\
\hline Total & 812 & 100,0 & 2.171 & 100,0 & 2.983 & 100,0 \\
\hline
\end{tabular}

1 Áreas mais desenvolvidas: Plano Piloto, Lagos Sul e Norte, Cruzeiro, Guará, Núcleo Bandeirante e Taguatinga.

2 Áreas menos desenvolvidas: São Sebastião, Brazilândia, Candangolândia, Ceilândia, Gama, Paranoá, Planaltina,

Samambaia, Sobradinho, Riacho Fundo, Santa Maria e Recanto das Emas.

mente maior de meninos, à semelhança do que ocorre na população. Por sua vez, a razão de sexos assemelha-se ao padrão esperado (Berquó, 1986). Os outros indicadores mostraram uma situação parecida a que seria esperada quando se comparam as regiões mais desenvolvidas com as menos desenvolvidas, como já assinalado. Isso ocorreu, por exemplo, com a taxa de parto cesariano, em acordo à encontrada em um estudo feito com dados secundários, em São Paulo, no período compreendido entre 1987 e 1993 (Rattner, 1996).
Evitar viés na aferição dos dados é fundamental para a credibilidade dos resultados de uma investigação. Neste inquérito, tais vieses foram neutralizados ou minimizados mediante a atenção dispensada à definição das variáveis investigadas e à forma de medi-las. Adotamos as definições de aleitamento materno recomendadas pela OPAS, estabelecidas por consenso, em reunião de especialistas (UNICEF et al., 1991; WHO/UNICEF, 1992). Os demais procedimentos empregados na fase de planejamento e de coleta dos dados, para controlar os 
Figura 1

Prevalência estimada (\%) das modalidades de aleitamento materno segundo idade da criança. Distrito Federal, 1994.

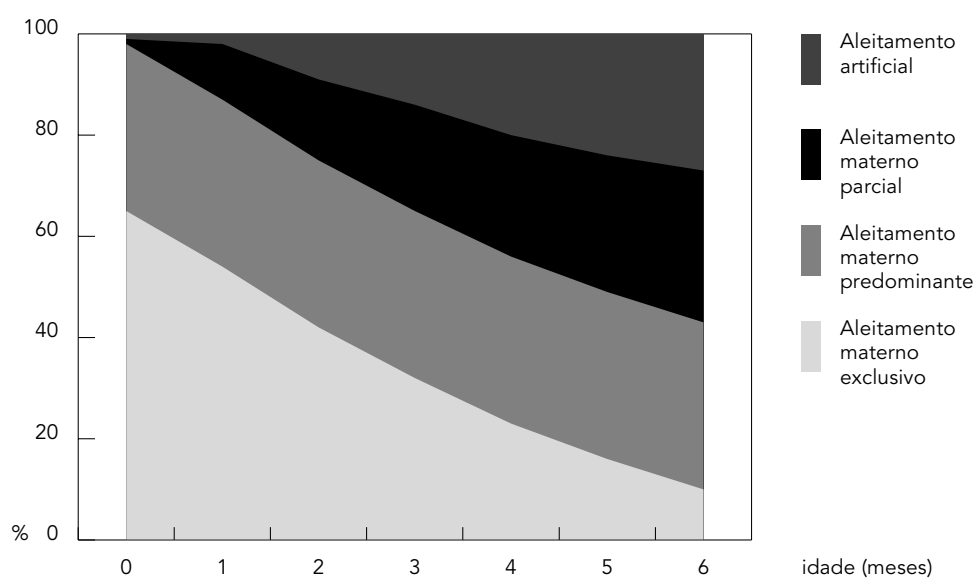

Nota: a prevalência de aleitamento artificial foi obtida por diferença

(100 - somatório das prevalências das três modalidades de aleitamento materno estimadas pela transformação logito). informação atual sobre a prática do aleitamento, ou seja, current breast-feeding status. Embora ela evite o erro relacionado à época da introdução de outros alimentos, depende de informação precisa sobre a data de nascimento da criança. A imprecisão desse dado, por sua vez, é menor quando o período decorrido entre o nascimento e a realização do inquérito é curto, e tende a ser atenuado em amostras grandes, situações essas contempladas no nosso estudo.

Não é do nosso conhecimento a existência de investigação anterior no Distrito Federal, para que os dados dessa pesquisa sejam comparados e saber se a situação está melhor, pior ou a tendência é estacionária. Tal comparação somente pode ser feita com resultados de estudos de base populacional, realizados em outras partes do país e que tenham observado determinados critérios metodológicos, entre os quais, a época de sua realização (três últimas décadas), tamanho adequado de amostra, técnica utilizada para a escol ha aleatória de seus elementos, intervalos etários comparáveis e método de investigação semelhantes.

A prevalência do aleitamento materno, no Distrito Federal, na faixa etária de 0 a 15 dias (96,8\%), foi maior que a encontrada em inquérito realizado no Município de São Paulo, de abril de 1984 a junho de 1985 (86,8\%) (Monteiro, 1988) e acima da freqüência da amamentação no Brasil (88\%), conforme a Pesquisa Nacional de Saúde e Nutrição, realizada em 1989 (Leão, 1992). Entretanto, tais comparações devem ser interpretadas com cautela. Primeiro, pelo tempo decorrido entre esses inquéritos e o do Distrito Federal. Segundo, pelas diferenças entre os limites etários, dentro do intervalo de 0 - 15 dias, analisadas nas três pesquisas. Terceiro, porque, mantendo-se as tendências de aumento do aleitamento natural, já observadas à época da realização das investigações mencionadas, é possível que, na atualidade, as diferenças em relação aos nossos dados sejam menores ou não mais existam. Além do mais, como a pesquisa foi realizada por amostragem, há sempre a possibilidade de um erro amostral em torno da prevalência, o que dificulta a interpretação de pequenas diferenças entre estudos.

Sobre o al eitamento exclusivo, a prevalência no Distrito Federal, no grupo de crianças com idade de 0 a 15 dias (62\%), foi menor que a estimada para o Município de São Paulo (77\%) (Monteiro, 1988) e para o Brasil (85\%) (Leão, 1992). A análise comparativa das estimativas de prevalência desse tipo de amamentação apresenta algumas limitações, sobretudo em relação aos estudos realizados antes de 1991, uma vez que, até aquela época, não havia con- 
Tabela 2

Prevalência estimada (\%) do aleitamento materno e suas modalidades, segundo a idade da criança e respectivos intervalos de confiança (IC 95\%). Distrito Federal, 1994.

\begin{tabular}{lcccr}
\hline $\begin{array}{l}\text { Intervalo } \\
\text { etário (dias) }\end{array}$ & $\begin{array}{l}\text { Ponto médio do } \\
\text { intervalo (dias) }\end{array}$ & $\begin{array}{l}\text { Aleitamento } \\
\text { materno1 } \\
\text { Prevalência (IC 95\%) }\end{array}$ & $\begin{array}{l}\text { Aleitamento } \\
\text { materno exclusivo } \\
\text { Prevalência (IC 95\%) }\end{array}$ & $\begin{array}{l}\text { Aleitamento } \\
\text { materno parcial } \\
\text { Prevalência (IC 95\%) }\end{array}$ \\
\hline $0-15$ & 7,5 & $96,8(95,8-97,7)$ & $62,0(58,5-65,3)$ & $4,5(3,1-6,4)$ \\
$16-30$ & 22,5 & $95,8(94,7-96,8)$ & $56,4(53,4-59,5)$ & $9,1(7,4-11,1)$ \\
$31-60$ & 45,0 & $93,9(92,7-95,0)$ & $47,9(45,4-50,3)$ & $13,9(12,3-15,6)$ \\
$61-90$ & 75,0 & $90,3(89,0-91,4)$ & $36,7(34,9-38,6)$ & $18,7(17,3-20,1)$ \\
$91-120$ & 105,0 & $85,3(83,9-86,6)$ & $26,8(25,1-28,6)$ & $22,4(20,9-24,1)$ \\
$121-150$ & 135,0 & $78,8(76,8-80,7)$ & $18,8(17,0-20,8)$ & $25,6(23,6-27,7)$ \\
$151-180$ & 165,0 & $70,9(67,7-73,9)$ & $12,8(11,0-14,7)$ & $28,4(25,8-31,0)$ \\
\hline
\end{tabular}

1 Aleitamento materno: crianças alimentadas no seio materno. Engloba as modalidades exclusivo, predominante e parcial (para as respectivas definições, ver texto).

A prevalência do aleitamento predominante manteve-se constante ao longo dos intervalos, estimando-se uma prevalência média de $33,1 \%(31,4-34,7)$.

Tabela 3

Distribuição do número de crianças na amostra, segundo o tipo de amamentação e a faixa etária. Distrito Federal, 1994.

\begin{tabular}{|c|c|c|c|c|c|c|}
\hline $\begin{array}{l}\text { Intervalos } \\
\text { etários } \\
\text { (dias) }\end{array}$ & $\begin{array}{l}\text { Ponto médio } \\
\text { dos intervalos } \\
\text { (dias) }\end{array}$ & $\begin{array}{l}\text { Número de } \\
\text { crianças no } \\
\text { intervalo }\end{array}$ & $\begin{array}{l}\text { Crianças em } \\
\text { aleitamento } \\
\text { materno }^{1}\end{array}$ & $\begin{array}{l}\text { Crianças em } \\
\text { aleitamento } \\
\text { exclusivo }\end{array}$ & $\begin{array}{l}\text { Crianças em } \\
\text { aleitamento } \\
\text { predominante }\end{array}$ & $\begin{array}{l}\text { Crianças em } \\
\text { aleitamento } \\
\text { parcial }\end{array}$ \\
\hline $0-15$ & 7,5 & 176 & 172 & 120 & 44 & 8 \\
\hline $16-30$ & 22,5 & 276 & 269 & 156 & 89 & 24 \\
\hline $31-60$ & 45,0 & 571 & 536 & 258 & 199 & 79 \\
\hline $61-90$ & 75,0 & 541 & 484 & 195 & 184 & 105 \\
\hline $91-120$ & 105,0 & 522 & 461 & 153 & 188 & 120 \\
\hline $121-150$ & 135,0 & 514 & 403 & 101 & 168 & 134 \\
\hline $151-180$ & 165,0 & 474 & 346 & 59 & 154 & 133 \\
\hline Total & NSA & 3.104 & 2.671 & 1.042 & 1.026 & 603 \\
\hline
\end{tabular}

NSA = não se aplica.

1 Engloba as modalidades de aleitamento materno exclusivo, predominante e parcial, mostradas nas três últimas colunas da tabela (para as respectivas definições, ver texto).

senso quanto à definição de conceitos para esse tipo de amamentação. Naquele período, alguns autores consideravam em al eitamento exclusivo as crianças amamentadas ao seio e às quais também se administrava água e chá; outros aceitavam, além da água e dos chás, o uso dos sucos, e ainda havia os que não explicitavam claramente de que forma seriam classificados esses lactentes. Assim, as diferenças observadas podem ser atribuídas às definições de termos, adotadas pelos três estudos em relação a essa forma de amamentação. Na investigação do Distrito Federal, considerou-se em al eita- mento exclusivo aquelas crianças que recebiam somente leite materno, sem uso de água, chás e sucos, conforme definição estabelecida em 1991 (UNICEF et al., 1991) e reafirmada em 1992, durante reunião de peritos promovida pela Organização Mundial da Saúde (OMS) e pelo UNICEF (WHO/UNICEF, 1992).

Além das definições das modalidades de amamentação, outro aspecto que, por vezes dificulta a comparação entre pesquisas, é a ausência de uniformidade dos intervalos etários analisados. Para tornar exeqüível a comparação entre os nossos dados e os de investigações 
que analisaram a situação do aleitamento exclusivo para outras faixas etárias, estimamos, também, a freqüência dessa forma de amamentação para as idades a serem comparadas. Desse modo, confrontando os nossos resultados com os de investigações epidemiológicas que empregaram a definição de aleitamento exclusivo, estabelecida na reunião de 1991, observa-se a situação aparentemente favorável do Distrito Federal quanto à prevalência dessa forma de amamentação. Com respeito a uma pesquisa realizada em Santos (São Paulo), em 1992 (Pérez-Escamilla, 1995), verificamos que, no Distrito Federal, 54\% das crianças com um mês de vida estavam em aleitamento exclusivo, enquanto que, naquela localidade, essa prevalência situava-se em $47 \%$. Em outra análise comparativa, feita com inquérito conduzido em Santo André (São Paulo), em 1991 (Ferreira, 1996), também se constata um quadro semeIhante. Na investigação do Distrito Federal, a prevalência da amamentação exclusiva, para os lactentes com 120 dias, foi de $23 \%$ e, para os com 180 dias, de $10 \%$, ao passo que, em Santo André, as freqüências foram de $14 \%$ e $4 \%$, respectivamente, para os mesmos grupos etários de crianças. Contudo, cabe ponderar que em relação à última localidade, essas diferenças podem ser devidas à população amostrada, visto que as mães entrevistadas foram selecionadas nas unidades de saúde, embora os autores chamem a atenção para a alta cobertura desses serviços, superior a $90 \%$.

Na pesquisa do Distrito Federal, estudamos também a duração mediana da amamentação exclusiva, que foi de 39,4 dias, número muito próximo ao de quarenta dias, encontrado no estudo de Santos (Pérez-Escamilla, 1995), superior à duração mediana de 28,9 dias, estimada para Santo André (Ferreira, 1996) e de 16,5 dias, para João Pessoa (Kitoko, 2000), mas inferior à duração mediana da amamentação ex- clusiva em Florianópolis, que foi de 53 dias (Kitoko, 2000). Esses números evidenciam a reduzida duração dessa forma de alimentação nas localidades mencionadas.

Procedendo-se análise semelhante para o aleitamento materno - que inclui as modalidades exclusivo, predominante e parcial - observa-se que, no Distrito Federal, para o grupo de crianças com 180 dias de vida, a prevalência dessa prática (60\%) é nitidamente superior à observada em Pelotas (Rio Grande do Sul), de 36,9\% (Victora, 1996) e, em Santo André, de 35,8\% (Ferreira, 1996). Tais valores configuram para as duas últimas localidades, um padrão de aleitamento, por ocasião da realização desses estudos, semelhante ao apresentado por alguns países desenvolvidos e pela elite urbana de algumas nações em desenvolvimento (OMS, 1981), refletindo a baixa prevalência e a curta duração dessa prática nas localidades investigadas.

A prevalência do aleitamento materno no Distrito Federal, mostra um padrão diferente do observado nas investigações mencionadas no parágrafo anterior. Na pesquisa aqui realizada, a prevalência da amamentação aos seis meses sugere a existência de um padrão intermediário entre, de um lado, as baixas freqüências encontradas em alguns países desenvolvidos e a elite urbana de algumas nações em desenvolvimento e, de outro, a alta prevalência do aleitamento materno em populações urbanas pobres e rurais de alguns países em desenvolvimento (OMS, 1981).

Em conclusão, os resultados do Distrito Federal fornecem um quadro da situação na metade da década de 90, que serve de base para o acompanhamento da prática do aleitamento materno. Embora se constate que a situação diagnosticada está longe da desejável, ela apresenta-se, aparentemente, um pouco melhor do que aquela verificada em algumas cidades e regiões brasileiras, utilizadas para comparações.

\section{Agradecimentos}

À Dra. Marly Vitali, co-responsável pelo planejamento e execução da pesquisa. Às Dras. Zuleika Portela Albuquerque, consultora em nutrição da Organização Pan-Americana da Saúde; Elvira Garcez de Castro Dória, coordenadora do Programa Nacional de Incentivo ao Aleitamento Materno à época de realização do estudo e Rosângela Conde Watanabe, diretora executiva da Fundação de Ensino e Pesquisa em Ciências da Saúde do Distrito Federal. Aos 49 supervisores, pela responsabilidade com que treinaram os entrevistadores e realizaram o trabalho de campo. Aos 503 alunos do segundo grau da Fundação Educacional do Distrito Federal, pelo entusiasmo e competência com que conduziram as entrevistas. 


\section{Referências}

AGRESTI, A., 1990. Categorical Data Analysis. New York: John Wiley.

BERQUÓ, E., 1986. Aleitamento materno diferencial para meninos e meninas. Cadernos de Pesquisa, 56:27-38.

COCHRAM, W. G., 1965. Técnicas de Amostragem. São Paulo: Editora Fundo de Cultura.

CUNNINGHAM, A. S., 1981. Breastfeeding and morbidity in industrialized countries: An update. In: Advances in International Maternal and Child Health (D. B. Jellife \& E. F. P. Jellife, ed.), pp. 128168, New York: Oxford University Press.

FEACHAM, R. G. \& KOBLINSKY, M. A., 1984. Interventions for the control of diarrhoeal diseases among young children: Promotion of breast-feeding. Bulletin of the World Health Organization, 62: 271-279.

FERREIRA, M. U.; CARDOSO, M. A.; SANTOS, A. L. S.; FERREIRA, C. S. \& SZARFARC, S. C., 1996. Rapid epidemiologic assessment of breastfeeding practices: Probit analysis of current status data. Journal of Tropical Pediatrics, 42:50-53.

HABICHT, J.-P.; DAVANZO, J. \& BUTZ, W. P., 1986. Does breastfeeding really save lives, or are apparent benefits due to biases? American Journal of Epidemiology, 123:279-290.

HORTA, B. L.; OLINTO, M. T.; VICTORA, C. G.; BARROS, F. C. \& GUIMARÃES, P. R. V., 1996. Amamentação e padrões alimentares em crianças de duas coortes de base populacional no sul do Brasil: Tendências e diferenciais. Caderno de Saúde Pública, 12(Sup. 1):43-48.

KITOKO, P. M.; RÉA, M. F.; VENÂNCIO, S. I.; VASCONCELOS, A. C. C. P.; SANTOS, E. K. A. \& MONTEIRO, C. A., 2000. Situação do aleitamento materno em duas capitais brasileiras: Uma análise comparada. Cadernos de Saúde Pública, 16:11111119.

LEÃO, M. M.; COITINHO, D. C.; RECINE, E.; COSTA, L. A. L. \& LACERDA, A. J., 1992. O perfil do aleitamento materno no Brasil. In: Perfil Estatístico de Crianças e Mães no Brasil: Aspectos de Saúde e Nutrição de Crianças no Brasil 1989 (M. F. G. Monteiro \& R. Cervini, org.), pp. 97-109, Rio de Janeiro: Fundação Instituto Brasileiro de Geografia e Estatística/ Instituto Nacional de Alimentação e Nutrição/ Fundo das Nações Unidas para a Infância.

MONTEIRO, C. A., 1988. Saúde e Nutrição das Crianças de São Paulo. São Paulo: Editora Hucitec.

MS (Ministério da Saúde), 1994. Em Busca do Cumprimento do Pacto pela Infância. Brasília: MS.
NOTZON, F., 1984. Trends in infant feeding in developing countries. Pediatrics, 74(Sup.):648-666.

OMS (Organización Mundial de la Salud), 1981. Modalidades de la Lactancia Natural en Ia Actualidad. Geneva: OMS.

PEREZ-ESCAMILLA, R.; LUTTER, C.; SEGALL, A. M.; RIVERA A.; TREVIÑO-SILLER, S. \& SANGHVI, T., 1995. Exclusive breast-feeding duration is associated with attitudinal, socioeconomic and biocultural determinants in three Latin American countries. Journal of Nutrition, 125:2972-2984.

RATTNER, D., 1996. Sobre a hipótese de estabilização das taxas de cesárea do Estado de São Paulo, Brasil. Revista de Saúde Pública, 30:19-33.

SAS INSTITUTE, 1990. Statistical Analysis System. Cary: SAS Institute.

UNICEF (Fundo das Nações Unidas para a Infâncias)/ USAID (United States Agency for International Development)/CDS (Programa de Censo Democrático de Saúde)/OMS (Organização Mundial da Saúde), 1991. Indicadores de Al eitamento Materno. Geneva: UNICEF/USAID/CDS/OMS. (mimeo.)

VICTORA, C. G.; BARROS, F. C.; HALPERN, R.; MENEZES, A. M.; HORTA, B. L.; TOMASI, E.; WEIDERPASS, E.; CÉSAR, J. A.; OLINTO, M. T.; GUIMARÃES, P. R. V.; GARCIA, M. M. \& VAUGHAM, J. P., 1996. Estudo longitudinal da população materno-infantil da região urbana do sul do Brasil, 1993: Aspectos metodológicos e resultados preliminares. Revista de Saúde Pública, 30:34-45.

VICTORA, C. G.; SMITH, P. G.; VAUGHAM , J. P.; NOBRE, L. C.; LOMBARD, C.; TEIXEIRA, A. M. B.; FUCHS, S. M. C.; MOREIRA, L. B.; GIGANTE, L. P. \& BARROS, F. C., 1987. Evidence for protection by breastfeeding against infant deaths from infections diseases in Brazil. Lancet, 2:319-322.

WHO (World Health Organization)/ UNICEF (United Nations Children's Fund), 1992. Indicators for Assessing Health Facility Practices that Affect Breastfeeding. Report of the Joint WHO/UNICEF Informal Interagency Meeting. Geneva: WHO/UNICEF. YOON, P. W.; BLACK, R. E.; MOULTON, L. \& BECKER, S., 1996. Effect of not breast feeding on the risk of diarrheal and respiratory mortality in children under two years of age in Metro Cebu, The Philippines. American Journal of Epidemiology, 143: 1142-1148.

Recebido em 16 de julho de 2001

Versão final reapresentada em 16 de setembro de 2001 Aprovado em 17 de dezembro de 2001 